\title{
Consolidado de recomendaciones del Comité Asesor de Vacunas y Estrategias de Inmunización-CAVEI, sobre priorización de vacunación COVID-19
}

\section{Consolidated recommendations of the Advisory Committee on Vaccines and Immunization Strategies- on prioritizing COVID-19 vaccination}

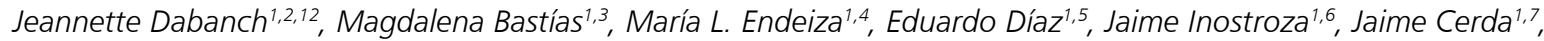 \\ Solange Santillana1,8, Jaime Rodríguez",9, Cecilia González,3, María Rodríguez'1,10 y Alejandra Pizarro ${ }^{1,11}$
}

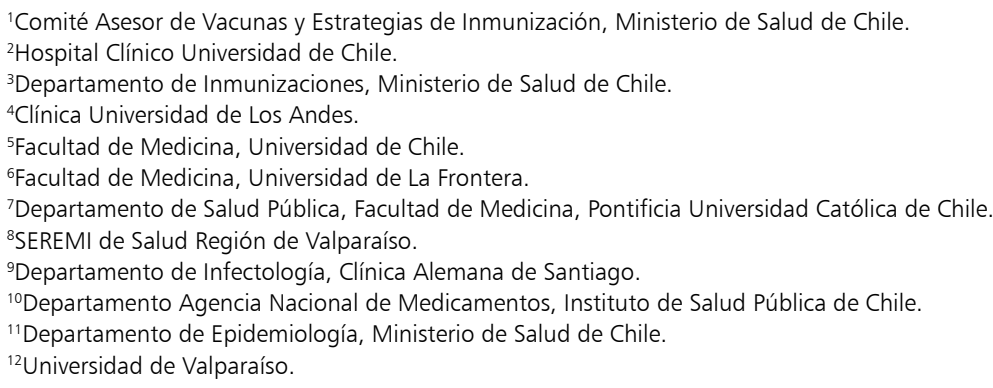

Recibido: 23 de marzo de 2021

\section{Resumen}

El COVID-19 es un problema de salud pública mundial por su carácter epidémico que, a la fecha, carece de tratamiento farmacológico. Sin embargo, ya se cuenta con algunas vacunas autorizadas para uso en emergencia, aunque la duración de su protección, su capacidad para interrumpir la transmisión viral y su eficacia frente a variantes emergentes de SARS-CoV-2 se encuentran en estudio. La campaña de vacunación contra SARS-CoV-2 de Chile requirió de diseño y planificación, como toda campaña. Parte de estos fue la priorización de grupos objetivo de vacunar, necesaria debido a que el mundo se vería enfrentado a un suministro limitado de vacunas COVID-19. En distintos momentos del año 2020, el CAVEI emitió recomendaciones sobre priorización de grupos de población a vacunar contra SARS$\mathrm{CoV}-2$, respondiendo a diferentes necesidades y según la evidencia disponible en cada instancia. Éstas se consolidan en la Tabla 1 de este informe. Resumidamente, en fase 1 se recomendó vacunar al personal de salud, residencias de larga estadía y personal crítico del Estado. En fase 2 , a personas mayores de 65 años y población con comorbilidades. En fase 3, a personas que cumplen labores esenciales y, finalmente, a la población general.

Palabras clave: SARS-CoV-2; COVID-19; vacunación masiva; campaña de inmunización; recomendaciones.

\section{Abstract}

COVID-19 is a global public health issue due to its epidemic nature that, to date, lacks pharmacological treatment. However, some COVID-19 vaccines have been authorized for emergency use, although the duration of their protection, their ability to interrupt viral transmission, and their efficacy against emerging variants of SARS-CoV-2 are being studied. Chile's SARS-CoV-2 vaccination campaign required design and planning, like any other campaign. This process included the prioritization of risk groups for vaccination given the limited supply of COVID-19 vaccines globally. Throughout 2020, CAVEI issued recommendations on the prioritization of population groups to be vaccinated against SARS-CoV-2 in response to different needs and in accordance with available evidence. These recommendations are consolidated in Table 1 in this report. In summary, it was recommended that healthcare workers, people in long-term residences and essential State personnel be vaccinated in phase 1 . In phase 2 , persons over 65 years of age and people with comorbidities. In phase 3, essential tasks workers and, lastly, the general population.

Keywords: SARS-CoV-2; COVID-19; vaccines; mass vaccination; immunization campaign; recommendations.

\section{Correspondencia a:}

Jeannette Dabanch Peña

jeannettedabanch@gmail.com 
$\mathrm{E}$ 1 COVID-19 es la enfermedad infecciosa causada por el coronavirus que se ha descubierto más recientemente, el SARS-CoV-2, en el brote en Wuhan, China, en diciembre de 2019. Los coronavirus son una extensa familia de virus que pueden causar enfermedades, tanto en animales como en humanos. En los humanos, se sabe que varios coronavirus causan infecciones respiratorias que pueden ir desde el resfrío común hasta enfermedades más graves como el síndrome respiratorio de oriente medio (MERS) y el síndrome respiratorio agudo severo (SARS).

El 30 de enero 2020, la Organización Mundial de la Salud declaró al brote de COVID-19 una emergencia de salud pública de importancia internacional (ESPII), en tanto el 11 de marzo de 2020 la declaró pandemia global ${ }^{1,2}$. A la semana epidemiológica 11 del 2021, Chile acumulaba 1.069 .461 casos (18 de marzo de 2021) y 29.540 defunciones (17 de marzo de 2021) por COVID-193.

El COVID-19 constituye un problema de salud pública mundial por su carácter epidémico que, a la fecha, carece de tratamiento farmacológico. Sin embargo, ya se cuenta con algunas vacunas autorizadas para uso en emergencia, aunque la duración de su protección, su capacidad para interrumpir la transmisión viral y su eficacia frente a variantes emergentes de SARS-CoV-2 se encuentran en estudio. Por tanto, las medidas epidemiológicas clásicas de control de diseminación del virus continúan siendo preponderantes.

La ejecución de la campaña de vacunación contra SARS-CoV-2, que en Chile comenzó en forma masiva el 3 de febrero de 2021, requirió de diseño y planificación, como ocurre con todas las campañas. Parte de esos procesos fue la priorización de grupos objetivo de vacunar, necesaria debido a que tanto Chile como el resto del mundo se vería enfrentado a un suministro limitado de vacunas COVID-19. Fue así como en distintos momentos de la pandemia, respondiendo a diferentes necesidades y en base a la evidencia e información disponibles en cada momento, el CAVEI emitió recomendaciones sobre priorización de grupos de población a vacunar contra SARS-CoV-2, a saber:
1. Pronunciamiento interino del CAVEI sobre grupos objetivo de vacunar contra SARS-COV-2, 30 de junio 2020.

https://vacunas.minsal.cl/wp-content/uploads/2020/07/ CAVEI_Pronunciamiento-Interino-GruposVacunacio\%CC\%81n-COVID19_30JUN2020.pdf

2. Recomendación provisoria del CAVEI para la priorización inicial de grupos a vacunar contra SARS-CoV-2 en el contexto de suministro limitado de vacunas, 2 de diciembre 2020.

https://vacunas.minsal.cl/wp-content/uploads/ 2020/12/CAVEI-Priorizacion-grupos-vacunaCOVID19_2Dic2020_FINAL-1.pdf.

3. Recomendaciones para priorizar la vacunación contra SARS-CoV-2 en personal de salud durante la fase de inicio de campaña y en el contexto de suministro limitado de vacunas, 15 de diciembre 2020.

https://vacunas.minsal.cl/wp-content/uploads/2020/12/ CAVEI-Subpriorizacion-personal-salud_15Dic2020. pdf.

4. Recomendación provisoria del CAVEI para la priorización inicial de grupos a vacunar contra SARS-CoV-2 en el contexto de suministro limitado de vacunas, enmienda 22 de diciembre 2020.

https://vacunas.minsal.cl/wp-content/uploads/2020/12/ CAVEI-Priorizacion-grupos-vacuna-COVID19_ENMIENDA-22dic2020_.pdf

5. Recomendaciones del CAVEI para priorizar la vacunación contra SARS.CoV-2 en personas mayores de 65 años en el contexto de suministro limitado de vacunas, 30 de diciembre 2020.

https://vacunas.minsal.cl/wp-content/uploads/ 2021/01/CAVEI-Subpriorizacion-personas-mayores 29Dic2020_final.pdf

Finalmente, la Tabla 1, a continuación, consolida las recomendaciones de priorización de vacunación COVID-19 emitidas por el CAVEI antes señaladas. 
Tabla 1. Consolidado de priorización de grupos objetivo de vacunar contra SARS-CoV-2 en Chile en el contexto de suministro limitado de vacunas COVID19. Se excluye a las mujeres embarazadast.

\begin{tabular}{|c|c|}
\hline $\begin{array}{l}\text { Prioridad } \\
\text { de acceso a } \\
\text { vacunación }\end{array}$ & Grupo poblacional \\
\hline \multicolumn{2}{|l|}{ Fase 1} \\
\hline $1 \mathrm{a}$ & $\begin{array}{l}\text { - Personal de salud clínico y administrativo en atención abierta, cerrada y urgencias, que incluye: servi- } \\
\text { cios clínicos (incluido dental), servicios de apoyo clínico (laboratorio, radiología, farmacia, anatomía } \\
\text { patológica), administrativos, alimentación, transporte, seguridad, aseo } \\
\text { - Estudiantes de carreras de las áreas de salud en práctica clínica }\end{array}$ \\
\hline $1 b$ & $\begin{array}{l}\text { - Personas residentes en centros de larga estadía, en centros del SENAME }{ }^{1} \text { o en convenio con esa } \\
\text { institución, en instituciones de atención de salud mental y personas privadas de libertad }\end{array}$ \\
\hline $1 c$ & $\begin{array}{l}\text { - Personal crítico² de la Administración del Estado } \\
\text { - Gendarmería } \\
\text { - Bomberos } \\
\text { - Fuerzas de Orden y Seguridad, Fuerzas Armadas desplegadas en el plan de acción por coronavirus }\end{array}$ \\
\hline \multicolumn{2}{|l|}{ Fase 2} \\
\hline $2 a$ & - Personas mayores de 65 años $^{3}$ \\
\hline $2 b$ & - Personas con co-morbilidades ${ }^{3-4}$ \\
\hline \multicolumn{2}{|l|}{ Fase 3} \\
\hline $3 a$ & $\begin{array}{l}\text { - Personal en labores de servicios básicos y esenciales }{ }^{5} \text {, tales como electricidad, agua, gas, generación } \\
\text { - Pe energía, combustibles, telecomunicaciones, recolección de desechos, rellenos sanitarios } \\
\text { - Personal de atención a público de FONASA y Registro Civil } \\
\text { - Personal de transporte de personas: metro, conductores de locomoción pública y ferrocarriles } \\
\text { - Personal de transporte de insumos críticos, como alimentos, insumos clínicos, medicamentos } \\
\text { - Personal crítico² de Municipios } \\
\text { - Personal crítico² de los } 23 \text { Ministerios de Estado } \\
\text { - Personal crítico² del Poder Judicial } \\
\text { - Personal } \mathrm{crítico}^{2} \text { del Poder Legislativo } \\
\text { - Personal } \mathrm{crítico}^{2} \text { de Gobiernos Regionales }\end{array}$ \\
\hline $3 b$ & $\begin{array}{l}\text { - Personal de salas cunas } \\
\text { - Personal de jardines infantiles } \\
\text { - Profesores de educación prescolar, básica y media }\end{array}$ \\
\hline $3 c$ & - Población general³ \\
\hline
\end{tabular}

tEn el caso de la vacuna COVID-19 de Pfizer autorizada para uso en emergencia por el Instituto de Salud Pública de Chile, éste ha señalado que la seguridad de la vacuna no ha sido probada en mujeres embarazadas ni en período de lactancia y que, por lo tanto, según la evidencia científica actual, no se recomienda su administración en mujeres gestantes ni mujeres en período de lactancia, agregando que a mujeres en período fértil se les recomienda evitar el embarazo por al menos dos meses luego de la vacunación. https://ispch.cl/sites/default/files/FIV\%20-\%20Coronavirus-24122020D.pdf. 1) Administración de vacuna COVID-19 a partir de la edad autorizada para su uso por el Instituto de Salud Pública de Chile. 2) Personal crítico: funcionarios que desarrollan labores necesarias para mantener el funcionamiento básico de la institución correspondiente y aquella que labora directamente en atención de público, en funciones de asistencia social o de apoyo directo a la ciudadanía. 3) Sujeto a la definición de sub-grupos beneficiarios según el tipo de tecnología de la vacuna disponible compatible con las características y necesidades de cada subgrupo. 4) Enfermedad pulmonar crónica (asma bronquial, EPOC, fibrosis quística, fibrosis pulmonar de cualquier causa); enfermedad neurológica (neuromusculares congénitas o adquiridas, que determinan trastornos de la deglución o del manejo de secreciones respiratorias, epilepsia refractaria a tratamiento); enfermedad renal crónica (insuficiencia renal en etapa 4 o mayor, diálisis); enfermedad hepática crónica (cirrosis, hepatitis crónica, hepatopatías); enfermedades metabólicas (diabetes mellitus, enfermedades congénitas del metabolismo); cardiopatías (congénitas, reumática, isquémica y miocardiopatías de cualquier causa); hipertensión arterial en tratamiento farmacológico; obesidad (IMC $\geq 30$ en adultos y en adolescentes IMC > +2 DE); enfermedad autoinmune (lupus eritematoso diseminado, esclerodermia, artritis reumatoide, enfermedad de Crohn, y otras); cáncer en tratamiento con radioterapia, quimioterapia, terapias hormonales o medidas paliativas de cualquier tipo; inmunodeficiencias (congénitas o adquiridas). 5) Servicios esenciales: distribuidores de combustible, elaboración de químicos y productos farmacéuticos, recolectores de basura y rellenos sanitarios, transporte de valores, suministro de energía y comercio exterior. 


\section{Referencias bibliográficas}

1.- World Health Organization. Novel Coronavirs (2019-nCoV) [Internet]. Situation Report 11. 2020 [cited 2020 May 20]. Available from: https://www. who.int/docs/default-source/coronaviruse/ situation-reports/20200131-sitrep-11-ncov. pdf?sfvrsn=de7c0f7 4 .

2.- World Health Organization. Coronavirus disease 2019 (COVID-19) [Internet]. Situation Report 51. 2020 [cited 2020 May 20]. Available from: https://www.who.int/docs/default-source/ coronaviruse/situation-reports/20200311-sitrep- 51-covid-19.pdf?sfvrsn=1ba62e 57 10.

3.- Ministerio de Salud de Chile. Informe Epidemiológico $\mathrm{N}^{\circ} 104$ Enfermedad por SARS-CoV-2 (COVID-19) Chile 19-03-2021 [Internet]. 2020. Disponible desde: https:// www.minsal.cl/wp-content/uploads/2021/03/ Informe-epidemiolo $\% \mathrm{CC} \% 81$ gico-104.pdf. 